\title{
Endometrial Adenosquamous Carcinoma
}

National Cancer Institute

\section{Source}

National Cancer Institute. Endometrial Adenosquamous Carcinoma. NCI Thesaurus.

Code C114656.

A rare endometrial carcinoma characterized by the presence of both malignant glandular and malignant squamous cellular components. 